\title{
Efficacy of Cognitive-Behavioral Therapy (CBT) on aggressive behavior in children with ADHD and emotion dysregulation: study protocol of a randomized controlled trial
}

Cécile Vacher ( $\nabla$ c-vacher@chu-montpellier.fr)

Centre Hospitalier Universitaire de Montpellier: Centre Hospitalier Regional Universitaire de Montpellier https://orcid.org/0000-0003-2794-3394

\section{Lucia Romo}

Universite Paris-Nanterre

Diane Purper-Ouakil

CHU Montpellier: Centre Hospitalier Regional Universitaire de Montpellier https://orcid.org/0000-00030454-1579

\section{Research Article}

Keywords: ADHD, Emotion Dysregulation, Children, Cognitive Behavioral Therapy

Posted Date: August 9th, 2021

DOl: https://doi.org/10.21203/rs.3.rs-446296/v1

License: (9) This work is licensed under a Creative Commons Attribution 4.0 International License.

Read Full License 


\section{Abstract}

\section{- Background:}

Attention Deficit Hyperactivity Disorder (ADHD) is frequently associated with emotional dysregulation (ED). ED is characterized by excessive and inappropriate emotional reactions compared to social norms, uncontrolled and rapid shifts in emotion, and attention focused on emotional stimuli.

Few studies have evaluated non-pharmacological interventions to improve ED in children with ADHD. The current randomized controlled trial assesses the efficacy of a Cognitive-Behavioral Therapy (CBT) intervention compared with a theater-based intervention (TBI) in children with ADHD and ED.

\section{- Methods:}

Sixty-eight 7 to 13-year-old children with ADHD and ED will be recruited and randomly assigned to the CBT or TBI group. CBT aims to reduce ED by teaching anger management strategies. TBI seeks to reduce ED by improving emotion understanding and expression through mimics and movement. In both groups, children participate in 15 one-hour sessions, and parents participate in 8 sessions of a parent management program. The primary outcome measure is the change in the "Aggression" sub-score of the Child Behavior Checklist (CBCL). Secondary outcome measures include overall impairment (Children's Global Assessment Scale, Strengths and Difficulties Questionnaire), personality profile (Hierarchical Personality Inventory for Children), executive function (Behavioral Rating Inventory of Executive Function), quality of life (Kidscreen-27), parental stress (Parenting Stress Index, $4^{\text {th }}$ edition), parental depression (Beck Depression Inventory-II), and impact of child disorders on the quality of the family life (Parental Quality of Life and Developmental Disorder).

\section{- Discussion:}

Children with ADHD and ED are at risk of functional impairment and poor outcomes, and have specific therapeutic needs. This randomized controlled trial wants to assess non-pharmacological treatment options for this population.

\section{- Trial registration:}

Clinicaltrials.gov. Number: NCT03176108. Registered on June 5, 2017

\section{Administrative Information}

\section{Note}

the numbers in curly brackets in this protocol refer to SPIRIT checklist item numbers. The order of the items has been modified to group similar items (see http://www.equator-network.org/reportingguidelines/spirit-2013-statement-defining-standard-protocol-items-for-clinical-trials/). 


\section{Title $\{1\} \quad$ Efficacy of Cognitive-Behavioral Therapy (CBT) on aggressive behavior in children with ADHD and emotion dysregulation: study protocol of a randomized controlled trial}

Trial registration $\{2 \mathrm{a}$

and $2 b\}$.

Clinicaltrials.gov. Number: NCT03176108. Registered on June 5, 2017

Protocol August 8, 2018; version n. 3

version $\{3\}$

Funding $\{4\} \quad$ The trial is funded by Montpellier University Hospital, France

Author details Vacher, $C^{1,2,3}$., Romo, $\mathrm{L}^{3,4,5}$., \& Purper-Ouakil ${ }^{1,2}, \mathrm{D}$.

$\{5 a\}$

${ }^{1}$ Centre Hospitalo-Universitaire de Montpellier, Service Médecine Psychologique de

l'Enfant et de l'Adolescent, Montpellier, Hérault, France

2 INSERM U 1018, CESP, Developmental Psychiatry/ADHD and emotional disorders, Montpellier, France

${ }^{3}$ CLIPSYD EA-4430, UFR Sciences Psychologiques et Sciences de l'Education, Université de Nanterre, Nanterre, Hauts de Seine, France

${ }^{4}$ CMME, Hôpital Sainte-Anne, GHU Paris Psychiatrie et Neurosciences

${ }^{5}$ INSERM UMR 1266, Institute of Psychiatry and Neuroscience, Paris.

Name and Vacher Cécile

contact

information for c-vacher@chu-montpellier.fr

the trial

sponsor $\{5 b\}$

Role of sponsor $\{5 \mathrm{c}\}$

The sponsor of this trial is Montpellier University Hospital and its team collects, analyzes and interprets the data; and writes the report in collaboration with Prof Romo. The decision to submit the report for publication comes from the principal research investigator.

\section{Introduction}

\section{Background and rationale $\{6 a\}$}

Attention-Deficit Hyperactivity Disorder (ADHD) is a complex neurodevelopmental disorder with a prevalence estimated at 5.3\% over the years (Polanzcyk \& al., 2007; Banaschewski \& al., 2017). ADHD is characterized by high levels of inattention, hyperactivity and impulsivity (APA, 2013) that have a lasting impact on the child and family's life.

Significant emotion dysregulation (ED) is observed in $24 \%$ to $50 \%$ children with ADHD (Shaw \& al., 2014; Qian \& al., 2016) and in 30\% to 70\% of adults with ADHD (Qian \& al., 2016). ED is the inability to modulate emotional responses in a given context (Thompson, 1994; Van Stralen, 2016), and includes: a) 
excessive and inappropriate emotional reactions and experiences relative to social norms; b) uncontrolled and rapid shifts in emotions; and c) abnormal allocation of attention to emotional stimuli (Shaw \& al., 2014). ED clinical expression is characterized by mood instability, irritability, aggression, tantrums (Masi \& al., 2015), and low tolerance to frustration (Wehmeier et al., 2010; McQuade \& Breaux, 2016; CaroCanizares \& al., 2017). Definitions of ED tend to focus only on negative emotions, but children with ADHD and ED can also experience difficulties in regulating positive emotions (D'Ambrogio \& Speranza, 2012; Sjöwall \& al., 2013). Physiological arousal (gritting teeth, upturned mouth, physical tension...) and cognitive biases ("Why is that person strangely looking at me?"; "He/she purposely annoys me") contribute to the socially inappropriate behaviors in patients with ED (Rosen \& al., 2018). As ED is present in many psychiatric disorders (e.g. anxiety disorders, mood disorders, neurodevelopmental disorders), it is not specific to ADHD (Bunford \& al., 2015; Stringaris \& al., 2018; Kircanski \& al., 2018).

Besides the core ADHD symptoms, children with ADHD and ED show higher levels of functional impairment (Rosen \& Factor, 2015; Walerius \& al., 2018), aggressive behaviors, oppositional defiant disorder, mood and anxiety disorders (Anastopoulos \& al., 2011; Vidal-Ribas \& al., 2016). Children with $A D H D$ and $E D$ use inappropriate coping strategies that interfere with their academic performance and social functioning (Walerius \& al., 2018). Therefore, ED contributes to their social and academic difficulties (Sobanski \& al., 2010; Surman, \& al., 2011; Bunford \& al., 2015).

Aggressive behaviors are one of the most problematic expressions of high ED (Anastopoulos \& al., 2011; Maire \& al., 2017). Aggression is defined as verbal and/or physical acts with the aim of harming another person, directly or indirectly (Ghanizadeh \& al., 2010). Proactive aggression is a deliberate goal-oriented behavior that is motivated by external reinforcements (Crick \& al., 1996; Bunford \& al., 2015). Reactive aggression is a violent reaction to ambiguous situations where the behavior of others tends to be interpreted as hostile and threatening (Crick \& al., 1996; D'Ambrogio \& al., 2012). Reactive aggression is frequent in $A D H D$ and is associated with difficulties of self-regulation, including ED and executive function deficits (White \& al., 2013; Bunford \& al., 2015; Murray \& al., 2016; Bartels \& al., 2018). Children with ADHD and high levels of aggression also show more inadequate emotional coping strategies compared with those with low levels of aggression (Melnick and Hinshaw, 2000).

Medications, such as psychostimulants and risperidone, improve aggressive behaviors, emotional dysregulation, and rageful outbursts (Blader \& al., 2013; Tourian \& al., 2014; Gadow \& al., 2014; Fernandez de la Cruz \& al., 2015; Kultu \& al., 2017; Winters \& al., 2018; Gamli \& al., 2018; Bruno \& al., 2019). It has been hypothesized that such drugs, by improving impulsivity, allow children with ADHD to better understand emotions and to plan appropriate strategies to cope with problematic situations (Gamli \& al., 2018).

Many studies have also focused on psychosocial interventions (parental management training, CBT...) of irritability, but not specifically in children with ADHD. Indeed, irritability (Stoddard \& al., 2016) and aggressive behaviors (Penton-Voak \& al., 2013) can be reduced by learning how to modify hostile interpretation bias and to improve emotion recognition. For instance, the aim of CBT programs for 
irritability and anger problems is to reduce hostile interpretations (Brotman \& al., 2017), to improve emotional regulation skills (Derella \& al., 2019), anger management (Thi Bui \& al., 2018), and the overall functioning of children (Kircanski \& al., 2018). A review of meta-analyses indicates that CBT programs are beneficial for children with anger problems (Hyoeun \& al., 2018) and aggression (Sukhodolsky et al., 2004). Moreover, a systematic review showed that psychosocial interventions have beneficial effects on emotional symptoms (irritability, depression), aggressiveness, and overall functioning in children with ADHD and ED (Vacher \& al., 2020). Several studies on emotional regulation in children with ADHD suggest that intervention allows reducing emotional problems and better regulating negative emotions (Herbert \& al., 2013; Hannesdottir \& al., 2017; Sanchez \& al., 2019). However, few studies specifically focused on the management of aggressive behavior and anger in children with both ADHD and ED, although these are the most important manifestation of ED. A combination of a self-control program and Anger Control Training reduces aggressive behavior in children with ADHD (Miranda and Presentacion, 2000). Masi et al., (2015) reported that in children with ED and disruptive behavior disorders (including $25 \%$ with ADHD), a multimodal treatment program led to a modest to significant improvement in the "Aggressive behavior" sub-scale of the Child Behavior Checklist (CBCL), depending on ED severity.

Battagliese et al., (2015) showed that CBT is effective for aggressive behaviors only when interventions are delivered to both parents and child. Similarly, most psychosocial interventions that are effective on ED also included a parent program (Herbert \& al., 2013; Fernandez de la Cruz \& al., 2015; Waxmonsky \& al., 2016; Hannesdottir \& al., 2017). A meta-analysis indicated that behavioral parent training is effective for reducing oppositional and aggressive behavior in children with ADHD (Fabiano \& al., 2015). Behavioral and cognitive-behavioral parent programs help parents to manage their child's behavior by teaching behavioral and cognitive-behavioral techniques (Zwi \& al., 2011). According to previous studies (Walerius \& al., 2018; Kircanski \& al., 2019), parents learn to identify the triggers of anger, to predict problematic behaviors, and to cope with their child's emotional reactions. Moreover, an emotion socialization program for parents allowed children with ADHD to develop emotional skills and had a protective effect towards comorbidities (Herbert \& al., 2013). As children learn emotional regulation strategies by observing their parents, the parents' role is crucial to help them to adjust their emotions and acquire emotional skills (Braet \& al., 2014).

Many studies have shown the positive effects of psychosocial interventions on ED, particularly on aggressive behavior and anger, but they have many limitations. Indeed, most studies were uncontrolled, used different measurements, and had small and heterogeneous samples (Saini \& al., 2009). As there is no validated treatment for ED (Jairam \& al., 2012), particularly for children with ADHD and ED, interventions to help children with ADHD and ED to recognize and cope with their emotional problems should be targeted to allow them to reduce the frequency and intensity of their emotional reactivity (Rosen and Factor, 2015).

This randomized trial compares the effects of a parent/child CBT program and a theater-based intervention (TBI) on aggressive behavior in children with ADHD and ED. This trial proposes an 
intervention to manage ED in children with ADHD and their parents, and include an active control group (TBI), differently from most randomized controlled trials (Vacher \& al., 2020).

\section{Objectives $\{7\}$}

The main objective of this study is to evaluate the effectiveness of a parent/child CBT program compared with a TBI on aggressive behavior in children with ADHD and ED, 6 months after the intervention end.

Secondary objectives are to evaluate the CBT impact on the child's socio-communicative capacities, quality of life, executive functions, and overall functioning and on parental stress at the intervention end (short-term effects) and at month 6 post-intervention.

The hypotheses of this study are:

1. The CBT program will lead to a statistically higher reduction of the "Aggressive behavior" sub-score compared with the TBI program;

2. The CBT program will improve the quality of life in children and their parents, overall functioning, as well as parental stress and depressive symptomatology;

3. The benefits of the CBT program will persist at 6 months post-intervention;

4. CBT will improve executive functions measured with the "Behavioral Control Index" of the Behavioral Rating Inventory of Executive Function (BRIEF) questionnaire.

\section{Trial design $\{8\}$}

The study described in this protocol is a randomized controlled trial to test the efficacy of a CBT program for children with ADHD and ED. Sixty-eight children with ADHD and ED will be randomized (1:1) in the CBT or TBI group. Randomization will be stratified by school level and by the psychopathological disorder severity using the Child Behavior Checklist-A-A-A (combination of "Aggression", "Anxiety/Depression" and "Attention" subscales). Children will be divided in groups according to their school level: primary school (younger than 11 years) and middle school (11-13 years of age). This classification is required because the situations encountered in everyday life are not the same in middle and primary school.

\section{Methods: Participants, Interventions And Outcomes}

\section{Study setting $\{9\}$}

Patients will be recruited at the child psychiatry and neuropediatric units of Montpellier University Hospital. Information leaflets are distributed by mental health professionals to families during consultations in the outpatient services. Full study information is given to parents and children by the investigator before the signature of the informed consent. 


\section{Eligibility criteria $\{10\}$}

Inclusion criteria:

Inclusion criteria are: 1) Child/adolescent aged between 7 and 13 years; 2) diagnosis of ADHD (based on the DSM-5 diagnostic criteria) assessed with a structured clinical interview (Kiddie-Schedule for Affective Disorders and Schizophrenia Present and Lifetime version; K-SADS-PL), with a supplement to assess the presence of Severe Mood Dysregulation; 3 ) a score $\geq 180$ at the CBCL-A-A-A when combining the "Aggression", "Anxiety/Depression" and "Attention" subscales; 4) child/adolescent followed at Montpellier University Hospital; and 5) families covered by the national healthcare insurance.

\section{Exclusion criteria:}

Exclusion criteria are: 1) child with delayed development or severe language disorder; 2) parents who do not speak French; 3 ) lack of informed consent and assent by the parents and child for their participation in the study; and 4) child not living with at least one parent.

Study exit criteria: 1) participants lost to follow-up; and 2) consent withdrawal by the parents. Each study exit will be explained and described in detail.

\section{Who will take the informed consent? $\{26 \mathrm{a}\}$}

Informed consent and assent are obtained from the parents and children, respectively, before starting any trial-specific procedure. All participants are advised that participation in research is entirely voluntary, and that they can withdraw their participation at any time. Families are not paid for their participation. The child's psychiatrist or pediatrician does the first presentation of the study. The protocol is then explained in detail by the clinical investigator before the signature of the informed consent by parents and child.

\section{Interventions}

\section{Explanation for the choice of comparators $\{6 b\}$}

In this trial, two presumably active groups are compared: the CBT group based on the program "How to improve anger and frustration management" ("Mieux gérer sa colère et ses frustrations », Massé \& al., 2012), and the TBI group in which a theater-based group activity is proposed to develop verbal and reciprocal communication, theory of mind, self-awareness of emotions and interpersonal trust (Glass \& al., 2000; Corbett \& al., 2017; Felsman \& al., 2019). TBI improves social skills, socio-cognitive functioning, and social interactions in children (Corbett et al., 2016). TBI also improves self-esteem and shows medium effects sizes for anxiety and depression reduction (Krueger \& al., 2019).

TBI was chosen as active comparison group, because the objective of our study is to evaluate the primary and secondary outcomes in two potentially effective groups, while controlling for non-specific effects. In both groups, conditions are similar: duration and number of sessions, group location and setting, recruitment procedures. Differences concern only on the program content (CBT versus TBI). 


\section{Intervention description $\{11$ a\}}

\section{Cognitive Behavioral Therapy group:}

The CBT group is based on the manualized program "How to improve anger and frustration management" ("Mieux gérer sa colère et ses frustrations", Massé \& al., 2012). The program consists of 1hour sessions (one per week; 15 in total) led by a psychologist and a caregiver (nurse or educator) both trained in the management of behavioral disorders and ADHD. They also have in-depth knowledge of CBT that they practice regularly.

During the program, children learn to identify situations that cause anger and frustration, but also techniques to manage negative emotions. Each session is organized as follows: reminder of the previous session by correction of the task done at home, presentation of the theme of the session with practice in the form of role-plays or exercises, presentation of inter-session tasks, and session evaluation. Children evaluate each session on the following criteria: interest and pleasure in the proposed activities, quality of the documents, ease and usefulness of the session in everyday life. Children must achieve a behavioral goal during the session. This challenge is individualized and focuses on each child's specific difficulties. At the end of the session, the child evaluates whether he has achieved the goal. If the challenge is reached, he/she obtains a reward. Children learn that every effort deserves a reward. Several studies have shown that children with ADHD are particularly sensitive to the reward system and have emotional aversion to delay (Banaschewski \& al., 2012).

During the program, children learn how to identify signals of anger at the body level, triggering situations, behaviors, and thoughts that appear when they are angry. Children also learn ways to diminish angry outbursts, to prevent their increase (e.g. through relaxation, activity practice, thinking about something else), and to create a repertory of solutions to solve problems. They learn to interpret social situations and the importance of thoughts in triggering and maintaining anger. During the final sessions, children learn techniques to peacefully solve conflicts with others and to deal with the anger of others, whether or not this outburst is justified.

\section{Theater-based Intervention (TBI):}

TBI is a role-play activity that is part of our day-care activity schedule. The TBI group participates in 1hour sessions (one per week; 15 in total) led by two mental health workers (nurse or special educator) trained in the management of behavioral disorders, $A D H D$, and experienced in theater-play. The TBI sessions are not based on a specific program, and their content was developed from existing acting techniques already used in psychiatry (Héril and Mégrier, 2005; Alix and Renard, 2015).

The program objectives are to develop self-control and self-confidence skills, pro-social skills, and appropriate expression of emotions through role-play exercises. During the program, children are also trained in other competences, such as impulsivity control.

Each session includes structured and tailored activities: 
- A warm-up phase around active group exercises: children are in motion, while learning how to regulate their impulsivity;

- An individual phase with exercises of improvisation, expression of emotions, and memory;

- A phase of game in pairs that includes several exercise types: role-playing around everyday life, games where children learn how to use pro-social skills and how to work in teams.

- A fun and collective play time that lasts 5 minutes. This time allows children to let off steam.

- A quiet time: at the session end, children enjoy a time of relaxation or meditation. This moment allows children to refocus on themselves and to learn techniques to manage their emotions. These exercises help to increase physical self-control that can promote better impulse control in children with ADHD (Glass \& al., 2000).

During each session, the program includes sensory games, movement/space games, imitation activities, self-control games, and emotion expression activities.

Each session is adapted according to the individual needs of the children in the group and their age.

\section{Parents Group:}

The parent program is the same in both groups because this study objective is to compare the two programs for children and to evaluate their effectiveness on aggressivity in the context of ADHD with ED. Parents' sessions take place at the same time as the children's sessions, for convenience.

The parent group is based on a parent training program in which parents are taught behavioral and cognitive-behavioral techniques to effectively manage their child's behavioral problems (Zwi et al., 2011). There are many manual programs, such as Webster-Stratton's Incredible Years program (Webster-Stratton, 2006) and "How to improve living with ADHD at home" ("Mieux vivre le TDAH à la maison", Massé \& al., 2011). Previous studies have shown that parent management training improves childhood behavior problems, allows the development of positive parenting competences, and reduces parental stress (Furlong \& al., 2012). Parents learn techniques to manage their child's tantrums through videos and information sheets based on the experiences described by other parents. One session was added to this program on psychoeducation about ADHD and ED (session 1). In sessions 2 and 3, parents learn techniques on how to reinforce positive behaviors in their child (e.g. token system, special moment, encouragement...). Often parents of children with ADHD and ED tend to have conflictual and negative relationships with their own child and put in place coercive systems. In sessions 4 and 5, parents receive psychoeducation on anger (e.g. how to identify signs of anger, triggers) and learn several techniques for outburst management (e.g. to ignore minor disruptive behavior, to encourage emotion verbalization by their child). In session 6, parents learn problem-solving techniques, and techniques that allow parents and child to calmly discuss about conflicts and find solutions that are acceptable to both of them. In session 7, parents are taught several techniques to help their child manage their frustration. In session 8 , a parent 
is invited to share a problem encountered in their daily life with their child. The other parents in the group can ask questions to analyze the problem. Then, solutions are proposed, and a solution that seems effective is tried at home. This session allows putting into practice the techniques learnt during the program.

Each session lasts approximately one hour and it's carried out every second week. Between sessions, parents are asked to implement the acquired techniques in their everyday life. At the next session, sometime is dedicated to discuss their implementation and the difficulties encountered in order to adjust the techniques to the individual needs.

\section{Criteria for discontinuing or modifying the allocated interventions $\{11 \mathrm{~b}\}$}

Criteria for discontinuing the allocated interventions are: 1) disruptive behavior disorder that does not allow the child to benefit from the intervention; 2 ) behavior not stabilized by drugs for children under treatment; and 3) aggressive behavior of one participant toward the others.

The protocol does not allow modifying the allocated intervention.

\section{Strategies to improve adherence to interventions $\{11 \mathrm{c}\}$}

To improve adherence to the intervention protocols, behavioral goals are defined to increase motivation. These challenges are individualized and focus on each child's specific difficulties. At the session end, children must evaluate whether they have achieved their challenge. If the challenge is reached, they obtain a reward. Children learn that every effort deserves a reward.

During the study, participants will be contacted by telephone if absent during two consecutive sessions to know the reasons of their absence. Between sessions, caregivers are available by telephone or email to answer any questions from the families.

\section{Relevant concomitant care permitted or prohibited during the trial $\{11 \mathrm{~d}\}$}

During the interventions, pharmacological treatments are permitted. Moreover, pharmacological treatments can be modified if they are no longer appropriate. It is possible to begin a drug treatment during the trial if behavioral symptoms become too severe and have a negative impact on the child's participation and implication in the group. The maintenance of other usual care components is authorized.

It is recommended that parents do not participate in other parental management training programs, because of similarities between the interventions proposed in the trial and those usually offered by care services.

\section{Provisions for post-trial care $\{30\}$}


A participant may exit the trial for the following reasons: severe behavioral problems not sufficiently stabilized to allow the participant to benefit from the program, strong opposition to participate in the group, and inadequate behaviors (physical or verbal aggression against other participants or caregivers) that interfere with the proper functioning of the group. When a child behaves inappropriately (opposition, verbal or physical aggression, provocation...) during a session, a discussion time is offered with the child, parents and caregivers. The objective is to find a solution together to solve the problem. This exchange may be enough to motivate the participant and stop the inappropriate behavior. Concomitantly, the child's psychiatrist will be contacted and informed of the situation. He/she might decide to meet the child to modify the pharmacological treatment and to re-motivate him/her. Nevertheless, if the problematic behaviors persist and affect the group, the child exit from the program is decided together with the child, the family and the psychiatrist.

\section{Outcomes $\{12\}$}

Primary and secondary outcomes will be evaluated in the two groups (TBI and CBT) at the inclusion visit (baseline), at the intervention end, and at month 6 after the end of the intervention. The primary judgment criterion is the variation of the "Aggressive behaviors" score of the CBCL between baseline and month 6 post-intervention. The initial evaluation will be on outcomes at month 6 post-intervention; because we hypothesize that the effectiveness of such programs is observed several months after their end. Usually, it takes time for children and parents to assimilate and routinely implement the new techniques and also to observe the intervention benefits in the daily life. This will allow also assessing whether the beneficial effects of the interventions are maintained in the long term.

Secondary judgement criteria are the score variation between baseline at the intervention end of the CBCL-A-A-A, CBCL (total score), Kidscreen-27, Parental Quality of Life and Developmental Disorder (ParDD-Qol), Children's Global Assessment Scale (C-GAS), Parenting Stress Index-Short Form, $4^{\text {th }}$ edition (PSI4-SF), Beck Depression Inventory-II (BDI-II), and Strengths and Difficulties Questionnaire (SDQ) and the SDQ-dysregulation profile (SDQ-DP). Secondary judgement criteria include also the variation between baseline and month 6 post-intervention of the CBCL-A-A-A score, total CBCL score, Kidscreen-27 scores, Par-DD-Qol scores, C-GAS score, BRIEF scores, PSI-4-SF scores, BDI-II score, and SDQ and the SDQ-DP scores.

Other data will be collected during the initial visit: presence or absence of psychiatric disorders in children using the K-SADS-PL diagnostic tool, socio-demographic data, anamnestic data, and medication history. Pharmacological treatments will be recorded at each visit because the introduction of a new drug or the modification of posology may influence the research outcomes. Indeed, ADHD usual treatment effectively reduces ED (Tourian \& al., 2014; Fernandez de la Cruz \& al., 2015; Kultu \& al., 2017; Gamli \& al., 2018; Winters \& al., 2018). The Hierarchical Personality Inventory for Children (HiPIC) will be completed at the end of the intervention to limit the duration of the initial visit.

\section{Participant timeline $\{13\}$}


Outcome measures are collected at pretreatment (T1), just after treatment end (T2), and at month 6 posttreatment (T3) (Table 1). Data for the primary and secondary outcomes will be collected at child psychiatry unit of Montpellier University Hospital (MPEA Saint Eloi). The child presence is not required for all the measures collected during these assessments.

Pre-treatment visit (T1):

Participants and their parents will participate in a visit to determine their eligibility (Enrollment, Table 1). Then, the T1 visit takes approximately 2 hours, because the diagnostic interview (K-SADS-PL) is carried out systematically with the parents to confirm the diagnosis of ADHD and the presence of comorbidities. During this visit, parents are interviewed by clinicians for clinical ratings of their child's overall functioning impairment (C-GAS). Children and parents will also complete self-report questionnaires (see Table 1). A questionnaire (SDQ-Teacher) is completed also by the child's teacher. This questionnaire is either given to parents who will directly transmit it to the teacher, or sent by mail.

Visit at treatment end (T2):

After the last session, questionnaires are sent to the parents for completing them at home and bring them back at the T2 visit. Children and parents will complete the same self-report questionnaires as at T1, but for the HiPIC and the satisfaction questionnaires (only at T2), and BRIEF (only at T1) (Table 1). During the T2 visit, parents are interviewed by a clinician to assess the child's overall functioning (C-GAS). This visit does not exceed 30 minutes. If needed, questionnaires can also be completed with the help of a researcher. The SDQ-Teacher questionnaire is again completed by the child's teacher

Visit at month 6 post-treatment (T3):

This follow-up visit determines whether the intervention effects persist at month 6 after the intervention end. Children and parents will be interviewed by a clinician to complete the C-GAS. Before the visit, they will complete the same self-report questionnaires as at T1 (at home) (Table 1). This visit takes approximately 30 minutes.

Table 1. Visits and data acquisition during the trial 


\begin{tabular}{|c|c|c|c|c|}
\hline \multirow[b]{2}{*}{ TIMEPOINT } & \multicolumn{4}{|c|}{ Study period } \\
\hline & Enrollment & $\begin{array}{c}\text { Before the } \\
\text { intervention (T1) }\end{array}$ & $\begin{array}{c}\text { At the } \\
\text { intervention end } \\
\text { (T2) }\end{array}$ & $\begin{array}{l}\text { Six months after the } \\
\text { intervention (T3) }\end{array}$ \\
\hline \multicolumn{5}{|l|}{ nnrollment: } \\
\hline :igibility criteria & $\mathrm{X}$ & & & \\
\hline hild Behavior Checklist (CBCL) & $\mathrm{X}$ & & & \\
\hline aformed consent & $\mathrm{X}$ & & & \\
\hline andomization & $\mathrm{X}$ & & & \\
\hline \multicolumn{5}{|l|}{ issessments: } \\
\hline tructured clinical interview (K-SADS-PL) & & $\mathrm{X}$ & & \\
\hline \multicolumn{5}{|l|}{ arent-rated measures } \\
\hline hild Behavior Checklist (CBCL) & & $\mathrm{X}$ & $\mathrm{X}$ & $\mathrm{X}$ \\
\hline itrengths and Difficulties Questionnaire (SDQ-Par) & & $\mathrm{X}$ & $\mathrm{X}$ & $\mathrm{X}$ \\
\hline $\begin{array}{l}\text { iehavioral Rating Inventory of Executive Function } \\
\text { BRIEF) }\end{array}$ & & $\mathrm{X}$ & & $\mathrm{X}$ \\
\hline Iierarchical Personality Inventory for Children (HiPIC) & & & $\mathrm{X}$ & \\
\hline idscreen-27 & & $\mathrm{X}$ & $\mathrm{X}$ & $\mathrm{X}$ \\
\hline $\begin{array}{l}\text { 'arental Quality of Life and Developmental disorder in } \\
\text { neir children (Par-DD-Qol) }\end{array}$ & & $\mathrm{X}$ & $\mathrm{X}$ & $\mathrm{X}$ \\
\hline 'arenting Stress Index $4^{\text {th }}$ edition (PSI-4-SF) & & $\mathrm{X}$ & $\mathrm{X}$ & $\mathrm{X}$ \\
\hline ieck Depression Inventory-II (BDI-II) & & $\mathrm{X}$ & $\mathrm{X}$ & $\mathrm{X}$ \\
\hline \multicolumn{5}{|l|}{ hild-rated measures } \\
\hline idscreen-27 & & $\mathrm{X}$ & $\mathrm{X}$ & $\mathrm{X}$ \\
\hline \multicolumn{5}{|l|}{ eacher-rated measures } \\
\hline trengths and Difficulties Questionnaire (SDQ-Teacher) & & $\mathrm{X}$ & $\mathrm{X}$ & $\mathrm{X}$ \\
\hline \multicolumn{5}{|l|}{ linician-rated measures } \\
\hline hildren's Global Assessment Scale (C-GAS) & & $\mathrm{X}$ & $\mathrm{X}$ & $\mathrm{X}$ \\
\hline \multicolumn{5}{|l|}{ iatisfaction questionnaires: } \\
\hline \multicolumn{5}{|l|}{ arent-rated measures } \\
\hline atisfaction questionnaire on the parent program & & & $\mathrm{X}$ & \\
\hline atisfaction questionnaire concerning the child program & & & $\mathrm{X}$ & \\
\hline \multicolumn{5}{|l|}{ hild-rated measures } \\
\hline iatisfaction questionnaire on the child program & & & $\mathrm{X}$ & \\
\hline
\end{tabular}

\section{Sample size $\{14\}$}

As ED in children with ADHD has been only recently studied, it is difficult to find literature data for calculating the number of subjects required (particularly for assessing the changes in the "Aggressive behaviors", "Anxiety/Depression" and "Attentional Problems" scores of the CBCL scale).

The study by Masi and colleagues (2015) reported a change in the "Aggressive Behavior" score of the $\mathrm{CBCL}$ from 68.3 to 65.5 in the context of the non-comparative evaluation of an intervention similar to the one assessed in the present trial. The standard deviation of the score at the baseline was 5.4.

By making the reasonable assumption of a slightly more effective intervention than what reported by Masi G. et al., (2015), a CBCL score of 65 in the intervention group and 69 in the control group, with a common standard deviation of 5.5, can be expected at month 6 post-intervention. To highlight such a difference with a power of $80 \%$ and an alpha risk of $5 \%, 31$ subjects per group need to be included. By 
increasing this number by $10 \%$ to take into account participants lost to follow-up, the total number of subjects to be included in the study is 68 (34 per group).

\section{Recruitment \{15\}}

Patients will be recruited at the Child Psychiatry and Neuropediatric units of Montpellier University Hospital. Information leaflets will be distributed by psychiatrists and may be posted electronically to families. Researchers will give presentations on this trial to the general public and mental health professionals.

\section{Assignment of interventions: allocation}

\section{Sequence generation $\{16 a\}$}

After verification of the eligibility criteria, family's information and consent signature, each participant will be randomized. Intervention allocation will be done using a computer-based random number generator. Randomization will be performed as soon as the number of participants is reached to start a session (one CBT group and one TBI group) with a 1:1 ratio.

Randomization will be stratified by school level (primary vs middle school) and severity of psychopathological disorders using the CBCL-A-A-A score. Children will be divided in groups according to their school level (<11 years, and 11-13 years of age).

The investigator in charge of the study assessments is blinded to the group allocation.

\section{Concealment mechanism \{16b\}}

Randomization is done by the Department of Medical Information of Montpellier University Hospital using the Capture System software (Clinsight).

\section{Implementation $\{16 c\}$}

If participants are interested in the trial, psychiatrists or pediatricians give information and enroll them. Then, participants are contacted by telephone by the investigator. The psychologist informs about the study, verifies that families are aware of the implications of participation in a trial, and proposes an appointment for the initial evaluation. She verifies that children are motivated and willing to participate in the trial. Indeed, often, parents register their child without asking their opinion, while it is important that they are involved and motivated in order to benefit from the intervention. After the initial evaluation, participants are assigned to an intervention group by the research coordinator after randomization.

\section{Assignment of interventions: Blinding}

\section{Who will be blinded $\{17 \mathrm{a}\}$}


The research psychologist who performs the baseline and outcome assessments will be blind to the treatment conditions. Due to the nature of the interventions, it will not be possible for the researchers who administer the intervention to remain blind. Researchers who administer the intervention will not be involved in the collection of the outcome data to avoid bias in measuring results.

Given the study design, parents and children are not blind and are informed about the group allocation after randomization.

\section{Procedure for unblinding if needed $\{17 \mathrm{~b}\}$}

There is no procedure for revealing a participant's allocated intervention during the trial, because it is not a protocol targeting a pharmacological treatment. Therefore, it did not seem necessary to provide circumstances for and an unblinding protocol.

\section{Data collection and management}

\section{Plans for assessment and collection of outcome data $\{18 \mathrm{a}\}$}

Outcome data will be collected by a research psychologist. Data will be verified by supervised psychology students to detect errors in the collected data. Finally, data will be verified by a methodologist who will process all statistical data.

\section{Primary outcome}

Child Behavior Checklist (CBCL; Achenbach \& Rescorla, 2001):

The CBCL is a 118-item parent-completed questionnaire to measure emotional and behavior problems in 4 to 18-year-old children in the past six months. Parents rate each item as 0 (not true), 1 (somewhat or sometimes true), or 2 (very true or often true). The CBCL includes eight syndrome scores:

Anxious/Depressed, Withdraw/Depressed, Somatic Problems, Social Problems, Thought Problems, Attention Problems, Rule Breaking, and Aggressive Behavior. A computer program calculates the T-scores for each scale. Raw scores are converted to gender- and age-standardized scores. A T-score of 50 indicates average functioning and standard deviation is every 10 points.

The objective of the primary outcome is to assess the effectiveness of the CBT program on the "Aggressive behavior" score (i.e. primary outcome) of the CBCL-A-A-A. The CBT program should allow reducing aggression.

The "Aggressive behavior" sub-score contributes to the CBCL-DP and "Deficient Emotional SelfRegulation" (CBCL-DESR). The two profiles are the sum of the CBCL "Anxious/Depressed", "Attention Problems" and "Aggressive Behavior" (A-A-A) syndrome T-scores (Achenbach and Rescorla, 2001). The CBCL-DESR is present when the sum of the CBCL A-A-A scores is $\geq 180$, but below 210 ( $T$-scores $>60$ ). The "Dysregulation Profile" is present when the sum of the CBCL A-A-A scores is $\geq 210$ (2 SD; T-scores >70). These profiles represent a continuum of the emotional and behavioral dysregulation severity 
(Biederman \& al., 2012; Masi \& al., 2015). They allow defining an ED phenotype and are a risk marker of a persisting deficit of self-regulation of emotion and behavior (Holtmann \& al., 2011). CBCL-DP and CBCLDESR allow identifying a group of children with disruptive behavior (Aitken et al., 2019) and at risk of severe dysfunction (Spencer \& al., 2011; Holtmann \& al., 2011; Elmaghrabi \& al., 2020). The CBCL-DP and CBCL-DESR profiles are associated with higher risk of comorbidities (Holtmann \& al., 2011; De Caluwé \& al., 2013; Masi \& al., 2015), hospitalization in psychiatry services (De Caluwé \& al., 2013), and development of inappropriate personality traits (Peyre \& al., 2015) in adulthood. The CBCL-DESR profile is a predictor of increased risk of opposition defiant disorder, conduct disorder, and anxiety disorders (Spencer \& al., 2011). The CBCL-DP predicts high risk of mood disorders during adolescence (Masi \& al., 2015).

\section{Secondary outcomes}

The trial includes several secondary outcome measures, and also the clinician ratings of impairment (CGAS). Moreover, parents complete questionnaires about their child to evaluate:

- Global functioning (SDQ, C-GAS)

- Executive functions (BRIEF)

- Personality traits (HiPIC)

- Quality of life (Kidscreen-27).

Parents also complete questionnaires to evaluate:

- Parental stress (PSI-4-SF)

- Parental depression (BDI-II)

- The impact of their child disorders on the quality of family life (Par-DD-Qol).

Secondary outcomes include also teacher-report measures of the child's global functioning (SDQTeacher), and child-report measure of the quality of life (Kidscreen-27).

The study objectives are to evaluate the CBT program impact on the socio-communicative capacities, emotional and behavioral self-regulation capacities, quality of life of child and family, overall functioning of child, and parental stress/depression in the short term (intervention end) and also at month 6 postintervention. Finally, the personality profiles of children with ADHD will be analyzed to determine whether there are common personality traits in children with ADHD and ED.

Outcome measures

French version of the Strengths and Difficulties Questionnaire (SDQ-Fr, Goodman, 1997): 
SDQ-Fr is a standardized questionnaire completed by parents or teachers that allows a brief emotional and behavioral screening of 2 to 17-year-old children and adolescents (http://www.sdqinfo.com). The SDQ-Fr includes 25 items divided in 5 subscales with 5 items/each to assess emotional symptoms, conduct problems, hyperactivity/inattention, peer relationship problems, and prosocial behavior. The questionnaire is completed on paper in 5-10 minutes.

The SDQ-Fr allows obtaining a "dysregulation profile" (SDQ-DP). It is a combination of two items from the Emotional symptom subscale ("Many worries, often seems worried", "Often unhappy, down-hearted or tearful"), two from the Conduct problem subscale ("Picked on or bullied by other children", "Steals from home, school or elsewhere"), and one from the Hyperactivity/Inattention subscale ("Restless, overactive, cannot stay still for long"). A study suggested that children with ADHD and SDQ-DP display more angry reactions and are less capable to control their anger (Caro-Canizares \& al., 2017). The SDQ-DP allows identifying children with ADHD at risk of severe difficulties in overall functioning. A study showed that SDQ-DP is a predictor of developing psychiatric disorders in the next 4 years (Wang \& al., 2017).

Kidscreen-27 (KIDSCREEN Group, 2006):

Kidscreen-27 is a questionnaire that evaluates the generic health-related quality of life (HRQol) in 6 to 18year-old children. The Kidscreen-27 items are derived from the Kidscreen-52 questionnaire. There are two versions, one completed by parents (or primary caregivers) and the other by children. Children evaluate the subjective perception of their wellbeing during the last week. Kidscreen-27 comprises five domains: "Physical well-being”, "Psychological well-being", "Autonomy and parents", "Social support and peers", and "School environment". The responses are scored on 5-point Likert scales for frequency (never to always), or intensity (not at all to extremely). Kidscreen-27 has been translated into 36 languages. Kidscreen-27 scores are converted to T-values (standardized mean=50, standard deviation=10). Higher scores indicate good HRQol and well-being (Ravens-Sieberer \& al., 2007).

Parental Quality of Life and Developmental Disorder in their children (Par-DD-Qol; Berdeaux, 1998):

The Par-DD-Qol is a parent-completed 17-item questionnaire to assess the impact of their child's chronic disabilities on the parental quality of life (Qol). It is particularly adapted to parents of children with neurodevelopmental disorders, because these are chronic conditions that impact the parents' Qol. The questionnaire includes several dimensions: "Emotional", "Daily Disturbance" and "Global Qol”. It is adapted from the Par-ENT-Qol (Berdeaux \& al., 1998) used in the general population with chronic ear, nose and throat (ENT) infections. Responses are scored on a 5-point Likert scale (not at all to very much). Several studies (Baghdadli \& al., 2014; Raysse, 2011) showed a reliability coefficient higher than 0.82 (Cronbach's alpha) for each dimension. A score below 40 indicates "no impact" on the parents' Qol, a score between 40 and 57 a "moderate impact", and a score higher than 57 a "high impact".

Behavioral Rating Inventory of Executive Function (BRIEF, Gioa, Guy \& Kentworthy, 2000): 
BRIEF is a parent- or a teacher-completed 86 -item inventory to assess the executive functioning of 5 to 18-year-old children at home and in school environments using a three-point scale for frequency (never to always). The instrument includes eight scales that measure executive functioning: initiate, work memory, plan/organize, organization of materials, and monitor (forming the metacognition index [MI]), and inhibit, shift and emotional control (forming the behavioral regulation index [BRI]). The BRI assesses the ability to use appropriate inhibitory control to shift cognitive sets and modulate emotions and behaviors. The MI assesses the ability to use working memory to initiate, plan and sustain future-oriented problem solving (McCandless \& al., 2007). BRIEF includes two validity scales (Negativity and Inconsistency of responses) to identify the parents' response styles and to validate the questionnaire quality. A T-score of 65 indicates clinically significant executive function impairment. Gioia et al., (2000) reported that the BRIEF reliability coefficient ranges from 0.80 to 0.97 for the two forms (Parent and Teacher), except for two scales ("Initiate" and "Shift").

Parenting Stress Index, $4^{\text {th }}$ edition, short form (PSI-4-SF, Abidin, 1983):

The PSI-4-SF is a 36-item parent-completed questionnaire to measure the parental stress and to detect difficulties in the parent-child dyad. This is defined as a state of psychological malaise in parents related to the parent-child relationship. Responses are provided on a 5-point Likert scale (totally disagree to totally disagree). The questionnaire includes three sub-scales ("Parental distress", "Dysfunction in parentchild interactions", and "Difficulties in children") to assess the factors that may influence the level of stress experienced by parents in their relationship with their child. The first subscale "Parental distress" allows measuring the distress experienced by parents in the exercise of their role. The second subscale "Dysfunction in parent-child interactions" measures the parents' satisfaction of their relationship with their child and whether the child meets their expectations. The third subscale "Difficulties in children" assesses the parents' degree of distress due to their child's difficult behavior. A score between the $85^{\text {th }}$ and $89^{\text {th }}$ percentile indicates high stress level, and a score $\geq 90^{\text {th }}$ percentile is clinically significant. Abidin (2012) reported a reliability coefficient of 0.84 (test-retest, 6 months) and internal consistency coefficient of 0.95 (Cronbach's alpha) for the total score. The three subscales have internal consistency coefficients of $0.90,0.89$ and 0.88 , respectively. PSI-4-SF is strongly correlated with the original version of the PSI-4, with a correlation coefficient of 0.98 for the Total Stress Scale.

Beck Depression Inventory-II (BDI-Il; Beck, Steer \& Brown, 1996)

The BDI-II is the most popular screening instrument for depression in adolescents and adults. It is a 21item self-report questionnaire that examines the behavioral, emotional, somatic and cognitive symptoms of depression in the past two weeks. Each item is rated on a 4-point Likert scale (ranging from 0 to 3 ), reflecting the symptom severity. Scores from 0 to 13 indicate "Minimal depression", scores from 14 to 19 "Mild depression", scores from 20 to 28 "Moderate depression", and scores higher than 29 indicate "Severe depression". 
Wang and Gorenstein (2013) reported a mean alpha coefficient of 0.9 (ranging from 0.83 to 0.96 ) and excellent coefficients of retest reliability (0.73 to 0.96$)$. The BDI-II is valid in different cultures and presents strong psychometric properties (Beck, Steer \& Brown, 1996).

Hierarchical Personality Inventory for Children (HiPIC, Mervielde \& De Fruyt, 1999):

HiPIC is a 144-item parent-completed questionnaire to obtain a profile of the personality of 6 to 12 -yearold children according to a five-factor model. Responses are scored on a 5-point Likert scale (from "Very untypical" to "Very typical"). Five personality dimensions are assessed: "Emotional Stability", "Extraversion”, "Benevolence”, "Conscientiousness", and "Imagination”. HiPIC is scored on 18 facets composed of 8 items/each that are summed and averaged to obtain the dimensions. The "Extraversion" dimension includes positive emotionality, energy in children, and ease in social situations. The "Emotional stability" dimension assesses negative emotions and reactions towards their environment. The "Conscientiousness" dimension measures the determination and drive to achieve a goal. The "Benevolence" dimension evaluates agreeableness, attitude in relationships, and ability to empathize. The "Imagination" dimension represents creativity, curiosity, and openness to new experiences. Mervielde and De Fruyt (1999) reported a reliability coefficient of 0.70 (Cronbach's alphas) for each domain and facet.

Children's Global Assessment Scale(C-GAS, Shaffer \& al., 1983):

C-GAS is a numeric scale to assess the general functioning in 4-16-year-old children. This scale is used and completed by mental health clinicians. A clinician interviews the child, parents and school staff to assess the child's global functioning. C-GAS is adapted from the Global Assessment Scale for adults. Scores are divided in ten categories that range from "Extremely impaired" (1-10) to "Doing very well" (91100).

Schedule for Affective Disorders and Schizophrenia for School-aged Children, Present and Lifetime version (K-SADS-PL; Kaufman \& al., 1997; French version Mouren-Siméoni \& al., 2002):

K-SADS-PL is a semi-structured diagnostic interview to assess all current and lifetime DSM-IV Axis I mental disorders in 6-18-year-old children. K-SADS-PL is administrated by trained mental health clinicians (master level). The additional module to measure severe mood disorders, according to the diagnostic criteria described by Leibenluft \& al., (2003), will also be used. The objective is to obtain more information on ED and its impact on daily life.

\section{Plans to promote participant retention and complete follow-up $\{18 \mathrm{~b}\}$}

Evaluation visits will be scheduled at the intervention end (T2) and at month 6 post-intervention (T3) (see Table 1). Two weeks before the intervention end, the psychologist will propose to parents an appointment for the T2 visit. For the appointment of the follow-up visit (T3), families will be contacted by telephone by the psychologist. For families difficult to reach, several telephone calls may be made. The child's referring psychiatrist will be also contacted to improve data collection (e.g. he/she might call the family to 
emphasize the importance of completing the questionnaires, refer family to the psychologist after consultation...).

For families unable to attend the visit (health problems...), it might be exceptionally decided to complete the questionnaires and the Case Report Form (CRF) during a telephone conversation. This will be indicated in the CRF.

If parents decide to interrupt their trial participation, the investigator will contact them to know whether they agree to complete the final questionnaires.

To promote participant retention during the program, families will be contacted by telephone in case of absence during two sessions.

\section{Data management $\{19\}$}

For each participant, data will be reported in a CRF, first in paper format and then electronically. The used software, Capture System, complies with the FDA recommendations on computerized systems for managing clinical trials.

The data manager will perform additional computerized consistency tests to detect the presence of nonstandard, missing, aberrant or incoherent data. These tests will be executed regularly during the participants' recruitment and monitoring. Each identified incoherence will be the subject of a request for clarification to the researcher.

Data will be saved by the Clinical Research and Epidemiology Unit (CREU) of Montpellier Hospital and will be stored in ASCII type format.

\section{Confidentiality $\{27\}$}

Data will be collected in the CRF only by the research psychologist. Each CRF will be anonymized to respect the participants' confidentiality.

\section{Statistical methods}

\section{Statistical methods for the primary and secondary outcomes $\{20 \mathrm{a}\}$}

An intention-to-treat approach will be used to analyze the primary and secondary data from the trial. Participants will be analyzed in their randomization arm and cannot change group during the study.

The primary outcome measure is the changes of the "Aggressive behavior" sub-score between T1 (baseline) and T3 (month 6 post-intervention). A Z test will be used to compare the changes of the primary (between T1 and T3) and secondary outcomes (between T1 and T2/T3) in the CBT group and TBI group. In the case of group non-comparability, a multivariate model by linear regression could be carried out to take into account the potential confounding bias. 


\section{Interim analyses $\{21 \mathrm{~b}\}$}

In this trial, statistical analyses of data will be realized when the statistician will have all data (i.e. when the number of subjects required will be reached and when all visits will have been carried out).

The final decision to finish the trial will be taken by mutual agreement of the principal investigator, the data manager, the research coordinator, and the independent safety monitor. Data will be locked when all data have been checked and all corrections done.

\section{Methods for additional analyses (e.g. subgroup analyses) \{20b\}}

In the case of non-comparability for one or more parameters, an adjustment will be made on this/these parameter(s) for analysis of the judgment criteria. A multivariate linear regression model might be performed to account for potential confounders.

The protocol does not plan analyses for additional subgroups.

\section{Methods in analysis to handle protocol non-adherence and any statistical methods to handle missing data $\{20 \mathrm{c}\}$}

Analysis of the primary and secondary outcomes will be carried out per protocol for all families who participated in two thirds of the intervention.

For missing data, under the hypothesis of a Missing at Random (MAR) or Missing Completely at Random (MCAR) mechanism, an imputation method using the Markov chain Monte-Carlo method will be used with 15 imputation cycles. The analysis will be performed independently for each complete database and the results will be taken into account for estimating the final parameters and their standard deviations.

\section{Plans to give access to the full protocol, participant level-data, and statistical code $\{31 \mathrm{c}\}$}

On request, participants will be informed about the trial overall results by the principal investigator. Families will not have access to personal data.

\section{Oversight and monitoring}

\section{Composition of the data monitoring committee, its role and reporting structure $\{21 \mathrm{a}\}$}

Data monitoring will be carried out by an independent safety monitor who will ensure the compliance with the trial regulatory aspects and will verify data collection. The safety monitor acts as a representative of the study promotor.

\section{Adverse event reporting and harms $\{22\}$}

Due to the intervention types, serious adverse events are not expected. Serious adverse events will be reported by the promotor only if they are directly related to the protocol. Each adverse event will be 
recorded in the CRF by the principal investigator and will be monitored until resolution or stabilization. The principal investigator will evaluate each event and its gravity and will contact the trial promotor.

\section{Frequency and plans for auditing trial conduct $\{23\}$}

Investigators accept to comply with the regulatory requirements of the promoter and the competent authority for a research audit. Audit may be carried out at any stage of the trial, from the protocol development to the publication of results and archival of the data used for the study.

\section{Plans for communicating important protocol amendments to relevant parties (e.g. trial participants, ethical committees) $\{25\}$}

All protocol modifications must be submitted to and validated by the Local Human Subject Protection Committee before their implementation. When the protocol modification is accepted by the committee, the research assistant ensures that the changes are implemented accordingly.

\section{Dissemination plans $\{31$ a $\}$}

All communication of results must receive the prior agreement of the principal investigator and promoter. Montpellier Hospital is the data owner and must be mentioned as the trial promoter. On request, participants will be informed about the trial overall results by the principal investigator.

\section{Discussion}

This randomized controlled trial wants to investigate whether a CBT program can improve aggressivity in children with ADHD and ED. To this aim, the trial compares the effects of two interventions (CBT and TBI) on emotional and behavioral components in children with ADHD and ED. A parent-management program is implemented in both groups. Secondary objectives are to examine the intervention impact on the child's socio-communicative capacities, quality of life, executive function and overall functioning at the end of the intervention and after six months. The last objective is to examine the intervention effects on parental stress, quality of life and depressive symptoms. This is one of the first studies that evaluate the shortand long-term efficacy of a psychosocial intervention on aggressive behavior in children with ADHD and ED. Most studies tend to assess effectiveness in the short term (i.e. at the end of the intervention or after three months). To our knowledge, no study on psychosocial interventions in children with ADHD and ED assessed the effects of such programs after six months.

The results from this trial will be useful for the management of children with ADHD and ED, because this type of interventions should allow children to develop emotional and behavioral self-regulation skills by learning techniques to manage anger (relaxation, resolution problems...). Indirectly, it is expected that the program will promote the development of prosocial skills, leading to a more harmonious relationships with peers and family. For parents, the trial objective is to improve their educative strategies, and also the quality of family life and relations. 
The primary limitation of the trial is the lack of tools to detect the intervention effects on ED in children with ADHD. Indeed, most of the available tools for ED evaluation (Bunford \& al., 2015), such as physiological, observational and neuropsychological measures, do not take into account the specificities of subjects with ADHD (Faraone \& al., 2018). Therefore, the sub-scale "Aggressive behavior" of the CBCLDP was selected as the primary outcome, because it allows evaluating the degree of severity of aggression in children and adolescents (Althoff, 2010) and is a good ED marker.

ADHD represents a public health problem, because it has a significant impact on the children and their family's daily life and represents an economic burden (Le \& al., 2014). Developing effective early psychosocial treatment options for children with ADHD and ED is particularly important in order to prevent developmental trajectories with poor prognosis (social difficulties, comorbidities, persistence of ADHD symptoms), and possibly to limit the need of pharmacological treatments.

\section{Trial Status}

Protocol version: August 8, 2018 Version $n^{\circ} 3$.

Date of recruitment: June, 2017

End of recruitment: September, 2020

The article was submitted after the end of recruitment and not at the beginning: with the co-authors, we favored the implementation of research (doctors' information for recruitment, animation of groups) as well as the writing of a background article that has been published. In addition, due to the pandemic, several participants of the last group dropped out of the study and therefore we plan to extend our recruitement (we are currently waiting for feed-back from the methodologist).

Research is currently underway as patients are still participating in groups. The article was submitted just before the last initially planned visit.

\section{Abbreviations}

ADHD: Attention Deficit Hyperactivity Disorder; CBT: Cognitive Behavioral Therapy; CRF: Case Report From; ED: Emotional Dysregulation; TBI: Theater-based Intervention.

\section{Declarations}

Competing interests:

The authors declare that they have no competing interests.

\section{Acknowledgements}


We are sincerely grateful for the commitment and hard work of our colleagues of Montpellier Hospital: Odile Courtial, Julie Delettrez, Julie Fonseca Cruz, Agnès Furlan, Allison Goujon, Bertrand Moussion, Catherine Noirot, and Chantal Verdelhan.

We would like to sincerely thank Line Massé, Catherine Verret, and Fabienne Boudreault who let us use their program.

We also thank all participants involved in this study.

\section{Authors' contributions $\{31 \mathrm{~b}\}$}

All authors contributed to the trial design. CV drafted the manuscript. All authors contributed to the manuscript review. All authors read and approved the final manuscript.

\section{Funding $\{4\}$}

Montpellier University Hospital is the sponsor of this trial who finances the study design, collection, analysis and interpretation of data.

The trial is carried out following the NIH Clinical Study Protocols (Clinicaltrials.gov. Identifier: NCT03176108).

The views expressed are those of the authors and not necessarily those of the funders.

A part of the publication fees is funded by Hospital University Montpellier and another comes from other university budgets.

\section{Availability of data and materials $\{29\}$}

Data will be available upon request to the investigator.

\section{Ethics approval and consent to participate $\{24\}$}

Ethical approval for the trial was granted by the Local Human Subject Protection Committee ("Comité de Protection des Personnes Sud Méditerranée I"; Reference: ID RCB 2016-A01382-49). The parents' informed consent will be obtained from all participants.

\section{Consent for publication $\{32\}$}

Not applicable.

\section{Competing interests $\{28\}$}

The authors declare that they have no competing interests.

\section{Authors' details}


${ }^{1}$ Centre Hospitalo-Universitaire de de Montpellier, Service Médecine Psychologique de l'Enfant et de l'Adolescent, Montpellier, Hérault, France

2 INSERM U 1018, CESP, Developmental Psychiatry/ADHD and emotional disorders, Montpellier, France

${ }^{3}$ CLIPSYD EA-4430, UFR Sciences Psychologiques et Sciences de l'Education, Université de Nanterre, Nanterre, Hauts de Seine, France

${ }^{4}$ CMME, Hôpital Sainte-Anne, GHU Paris Psychiatrie et Neurosciences

${ }^{5}$ INSERM UMR 1266, Institute of Psychiatry and Neuroscience, Paris

\section{Authors' information (optional)}

\section{References}

1. Abidin RR. Parenting stress index [Spanish, French and Portuguese translations]. Lutz: PAR; 1983.

2. Abidin RR. Parenting stress index professional manual. 4th ed. Lutz: PAR; 2012.

3. Achenbach TM, Rescorla LA. Manual For the ASEBA School-Age Forms \& Profiles. Burlington: University of Vermont, Research Center for Children, Youth \& Families; 2001.

4. Aitken M, Battaglia M, Marino C, Mahendran N, Andrade BF. Clinical utility of the CBCL Dysregulation Profile in children with disruptive behavior. J Affect Disord. 2019;253:87-95. https://doi.org/10.1016/j.jad.2019.04.034.

5. Alix C, Renard I 100 exercices d'entraînement au théâtre, à partir de 8 ans. Retz, 2015.

6. Althoff RR, Verhulst FC, Rettew DC, Hudziak JJ, Van der Ende J. Adult Outcomes of Childhood Dysregulation: A 14-year Follow-up Study. Journal of the American Academy of Child Adolescent Psychiatry. 2010;49(11):1105-16.e1. https://doi.org/10.1016/j.jaac.2010.08.006.

7. American Psychiatric Association. Diagnostic and statistical manual of mental disorders. 5th ed. Arlington: American Psychiatric Publishing; 2013.

8. Anastopoulos AD, Smith TF, Garrett ME, Morrissey-Kane E, Schatz NK, Sommer JL, Kollins SH, Ashley-Koch A. Self-Regulation of Emotion, Functional Impairment, and Comorbidity Among Children With AD/HD. Journal of Attention Disorders. 2011;15:583592. https://doi.org/10.1177/1087054710370567.

9. Banaschewski T, Becker K, Döpfner M, Holtmann M, Rösler M, Romanos M. AttentionDeficit/Hyperactivity Disorder: A Current Overview. Deutsches Aerzteblatt Online. 2017. https://doi.org/10.3238/arztebl.2017.0149.

10. Banaschewski T, Jennen-Steinmetz C, Brandeis D, Buitelaar JK, Kuntsi J, Poustka L, Sergeant JA, Sonuga-Barke EJ, Frazier-Wood AC, Albrecht B, Chen W, Uebel H, Schlotz W, van der Meere JJ, Gill M, Manor I, Miranda A, Mulas F, Oades RD, ... Asherson P. Neuropsychological correlates of emotional 
lability in children with ADHD: Neuropsychological correlates of emotional lability. J Child Psychol Psychiatry. 2012;53(11):1139-48. https://doi.org/10.1111/j.1469-7610.2012.02596.x.

11. Baghdadli A, Pry R, Michelon C, Rattaz C. Impact of autism in adolescents on parental quality of life. Quality of life research. 2014;23(6):1859-68.

12. Bartels M, Hendriks A, Mauri M, Krapohl E, Whipp A, Bolhuis K, Conde LC, Luningham J, Ip F, Hagenbeek H, Roetman F, Gatej P, Lamers R, Nivard A, van Dongen M, Lu J, Middeldorp Y, van Beijsterveldt C, Vermeiren T. R., ... Boomsma, D. I. Childhood aggression and the co-occurrence of behavioural and emotional problems: Results across ages 3-16 years from multiple raters in six cohorts in the EU-ACTION project. European Child Adolescent Psychiatry. 2018. https://doi.org/10.1007/s00787-018-1169-1.

13. Battagliese G, Caccetta M, Luppino OI, Baglioni C, Cardi V, Mancini F, Buonanno C. Cognitivebehavioral therapy for externalizing disorders: A meta-analysis of treatment effectiveness. Behaviour Research Therapy. 2015;75:60-71. https://doi.org/10.1016/j.brat.2015.10.008.

14. Beck AT, Steer RA, Brown GK. Manual for the Beck Depression Inventory-II. San Antonio: Psychological Corporation; 1996.

15. Berdeaux G, Hervié C, Smajda C, Marquis P. Parental quality of life and recurrent ENT infections in their children: Development of a questionnaire. Quality of Life Research. 1998;7(6):501-12. https://doi.org/10.1023/A:1008874324258.

16. Biederman J, Petty CR, Day H, Goldin RL, Spencer T, Faraone SV, Surman CBH, Wozniak J. Severity of the Aggression/Anxiety-Depression/Attention Child Behavior Checklist Profile Discriminates Between Different Levels of Deficits in Emotional Regulation in Youth With Attention- Deficit Hyperactivity Disorder. Journal of Developmental Behavioral Pediatrics. 2012;33(3):236-43. https://doi.org/10.1097/DBP.0b013e3182475267.

17. Blader JC, Pliszka SR, Kafantaris V, Sauder C, Posner J, Foley CA, Carlson GA, Crowell JA, Margulies DA. Prevalence and Treatment Outcomes of Persistent Negative Mood Among Children with Attention-Deficit/Hyperactivity Disorder and Aggressive Behavior. Journal of Child Adolescent Psychopharmacology. 2013;26:164173. https://doi.org/10.1089/cap.2015.0112.

18. Braet C, Theuwis L, Durme K, Vandewalle J, Vandevivere E, Wante L, Moens E, Verbeken S, Goossens L. Emotion Regulation in Children with Emotional Problems. Cognitive Therapy Research. 2014;38(5):493-504.

19. Brotman MA, Kircanski K, Stringaris A, Pine DS, Leibenluft E. Irritability in Youths: A Translational Model. Am J Psychiatry. 2017;174:520-32. https://doi.org/10.1176/appi.ajp.2016.16070839.

20. Bruno A, Celebre L, Torre G, Pandolfo G, Mento C, Cedro C, Zoccali RA, Muscatello MR. A. Focus on Disruptive Mood Dysregulation Disorder: A review of the literature. Psychiatry Res. 2019. https://doi.org/10.1016/j.psychres.2019.05.043.

21. Bunford N, Evans SW, Wymbs F. ADHD and Emotion Dysregulation Among Children and Adolescents. Clinical Child Family Psychology Review. 2015;18(3):185-217. https://doi.org/10.1007/s10567-0150187-5. 
22. Bunford N, Evans SW, Becker SP, Langberg JM. Attention-Deficit/Hyperactivity Disorder and Social Skills in Youth: A Moderated Mediation Model of Emotion Dysregulation and Depression. Journal of Abnormal Child Psychology. 2015;43(2):283-96. https://doi.org/10.1007/s10802-014-9909-2.

23. Caro-Cañizares I, Serrano-Drozdowskyj E, Pfang B, Baca-García E, Carballo JJ. SDQ Dysregulation Profile and Its Relation to the Severity of Psychopathology and Psychosocial Functioning in a Sample of Children and Adolescents With ADHD. Journal of Attention Disorders. 2017: 1-8. https://doi.org/10.1177/1087054717691829.

24. Corbett BA, Blain SD, loannou S, Balser M. Changes in anxiety following a randomized control trial of a theatre-based intervention for youth with autism spectrum disorder. Autism. 2017;21(3):333-43. https://doi.org/10.1177/1362361316643623.

25. Corbett BA, Key AP, Qualls L, Fecteau S, Newsom C, Coke C, Yoder P. Improvement in Social Competence Using a Randomized Trial of a Theatre Intervention for Children with Autism Spectrum Disorder. Journal of Autism Developmental Disorders. 2016;46(2):658-72. https://doi.org/10.1007/s10803-015-2600-9.

26. Crick NR, Dodge KA. Social Information-Processing Mechanisms in Reactive and Proactive Aggression. Child Development. 1996;67:993-1002.

27. D’Ambrogio T, Speranza M. Approche psychopharmacologique des troubles du comportement chez l'enfant et l'adolescent. Neuropsychiatrie de l'Enfance et de l'Adolescence. 2012;60:5261. https://doi.org/10.1016/j.neurenf.2011.10.010.

28. De Caluwé E, Decuyper M, De Clercq B. The child behavior checklist dysregulation profile predicts adolescent DSM-5 pathological personality traits 4 years later. European Child Adolescent Psychiatry. 2013;22(7):401-11. https://doi.org/10.1007/s00787-013-0379-9.

29. Derella OJ, Johnston OG, Loeber R, Burke JD. CBT-Enhanced Emotion Regulation as a Mechanism of Improvement for Childhood Irritability. Journal of Clinical Child Adolescent Psychology 2019; 48(sup1): S146S154. https://doi.org/10.1080/15374416.2016.1270832.

30. Elmaghrabi S, Nahmias MJ, Adamo N, Di Martino A, Somandepalli K, Patel V, McLaughlin A, De Sanctis V, Castellanos FX. Is Increased Response Time Variability Related to Deficient Emotional Self-Regulation in Children With ADHD? Journal of Attention Disorders. 2020;24(7):1045-56. https://doi.org/10.1177/1087054718788950.

31. Fabiano GA, Schatz NK, Aloe AM, Chacko A, Chronis-Tuscano. A. A Systematic Review of MetaAnalyses of Psychosocial Treatment for Attention-Deficit/Hyperactivity Disorder. Clin Child Fam Psychol Rev. 2015;18(1):77-97. https://doi.org/10.1007/s10567-015-0178-6.

32. Faraone SV, Rostain AL, Blader J, Busch B, Childress AC, Connor DF, Newcorn JH. Practitioner Review: Emotional dysregulation in attention-deficit/hyperactivity disorder - implications for clinical recognition and intervention. J Child Psychol Psychiatry. 2018. https://doi.org/10.1111/jcpp.12899.

33. Felsman P, Seifert CM, Himle JA. The use of improvisational theater training to reduce social anxiety in adolescents. The Arts in Psychotherapy. 2019;63:111-7. https://doi.org/10.1016/j.aip.2018.12.001. 
34. Fernández de la Cruz, L, Simonoff E, McGough JJ, Halperin JM, Arnold EL, Stringaris A. Treatment of Children With Attention-Deficit/Hyperactivity Disorder (ADHD) and Irritability: Results From the Multimodal Treatment Study of Children With ADHD (MTA). Journal of the American Academy of Child Adolescent Psychiatry. 2015;54:62-70.

35. Furlong M, McGilloway S, Bywater T, Hutchings J, Smith SM, Donelly M. Behavioural and cognitivebehavioural group-based parenting programmes for early-onset conduct problems in children aged 3 to 12 years. Evid-Based Child Health. 2012;2:318-692. https://doi.org/10.1002/ebch.1905.

36. Gadow KD, Arnold EL, Molina BS, Findling RL, Bukstein OG, Brown NV, McNamara NK, RundbergRivera VE, Li X, Kipp H, Schneider J, Farmer CA, Baker J, Sprafkin J, Rice RR, Bangalore SS, Butter EM, Buchan-Page KA, Hurt EA, Austin AB, Grondhuis SN, Aman MG. Risperidone Added to Parent Training and Stimulant Medication: Effects on Attention-Deficit/Hyperactivity Disorder, Oppositional Defiant Disorder, Conduct Disorder, and Peer Aggression. Journal of the American Academy of Child Adolescent Psychiatry. 2014;53(9):948-59.e1. https://doi.org/10.1016/j.jaac.2014.05.008.

37. Gamli IS, Tahiroglu AY. Six Months Methylphenidate Treatment Improves Emotion Dysregulation in Adolescents with Attention Deficit/Hyperactivity Disorder: A Prospective Study. Neuropsychiatric Disease Treatment. 2018;14:13291337. https://doi.org/10.2147/NDT.S164807.

38. Ghanizadeh A, Haghighi HB. How do ADHD children perceive their cognitive, affective, and behavioral aspects of anger expression in school setting? Child Adolescent Psychiatry Mental Health. 2010; 4 (1). https://doi.org/10.1186/1753-2000-4-4.

39. Gioia GA, Isquith PK, Guy SC, Kenworthy L. Behavior Rating Inventory of Executive Function: Professionnal manual. Lutz: Psychological Assessment Resources; 2000.

40. Glass KL, Guli LA, Semrud-Clikeman M. Social Competence Intervention Program: A pilot Program for the Development of Social Competence. Journal of Psychotherapy in Independent Practice. 2000;1(4):21-33.

41. Goodman R. The Strengh and Difficulties Questionnaire: a research note. Journal of Child Psychological Psychiatry. 1997;38:581-6.

42. Hannesdottir DK, Ingvarsdottir E, Bjornsson A. The OutSMARTers Program for Children With ADHD: A Pilot Study on the Effects of Social Skills, Self-Regulation, and Executive Function Training. Journal of Attention Disorders. 2017;21(4):353364. https://doi.org/10.1177/1087054713520617.

43. Herbert SD, Harvey EA, Roberts JL, Wichowski K, Lugo-Candelas Cl. A Randomized Controlled Trial of a Parent Training and Emotion Socialization Program for Families of Hyperactive Preschool-Aged Children. Behav Ther. 2013;44:302-16.

44. Héril A, Mégrier D 60 exercices d'entraînement au théâtre, à partir de 8 ans, Vol.2. Retz 2005.

45. Holtmann M, Buchmann AF, Esser G, Schmidt MH, Banaschewski T, Laucht M. The Child Behavior Checklist-Dysregulation Profile predicts substance use, suicidality, and functional impairment: A longitudinal analysis: CBCL-DP long-term outcome. J Child Psychol Psychiatry. 2011;52(2):139-47. https://doi.org/10.1111/j.1469-7610.2010.02309.x. 
46. Jairam R, Prabhuswamy M, Dullur P. Do we really know how to treat a child with bipolar disorder or one with severe mood dysregulation? Is there a magic bullet? Depression Research Treatment. 2012. https://doi.org/10.1155/2012/967302.

47. Kaufman J, Birmaher B, Brent D, Rao U, Flynn C, Moreci P, Williamson D. \& Ryan N. Schedule for Affective Disorders and Schizophrenia for School-Age Children-Present and Lifetime Version (KSADS-PL): Initial Reliability and Validity Data. Journal of the American Academy of Child Adolescent Psychiatry. 1997;36(7):980-8.

48. Kircanski K, Clayton ME, Leibenluft E, Brotman MA. Psychosocial Treatment of irritability in Youth. Cur Treat Options Psychiatry. 2018;5(1):129-40. https://doi.org/10.1007/s40501-018-0141-5.

49. Kircanski K, Craske MG, Averbeck BB, Pine DS, Leibenluft E, Brotman MA. Exposure therapy for pediatric irritability: Theory and potential mechanisms. Behaviour Research Therapy. 2019;118:1419. https://doi.org/10.1016/j.brat.2019.04.007.

50. Krueger KR, Murphy JW, Bink AB. Thera-prov: A pilot study of improv used to treat anxiety and depression. Journal of Mental Health (Abingdon England). 2019;28(6):621-6. https://doi.org/10.1080/09638237.2017.1340629.

51. Kutlu A, Ardic UA, Ercan ES. Effect of Methylphenidate on Emotional Dysregulation in Children With Attention-Deficit/Hyperactivity Disorder + Oppositional Defiant Disorder/Conduct Disorder. Journal of Clinical Psychopharmacology. 2017;37:220225. https://doi.org/10.1097/JCP.0000000000000668.

52. Le HH, Hodgkins P, Postma MJ, Kahle J, Sikirica V, Setyawan J, Erder MH, Doshi JA. Economic impact of childhood/adolescent ADHD in a European setting: the Netherlands as reference case. Eur Child Adolesc Psychiatry. 2014;23:587-98. https://doi.org/10.1007/s00787-013-0477-8.

53. Leibenluft E, Charney DS, Towbin KE, Bhangoo RK, Pine DS. Defining clinical phenotypes of juvenile mania. The American Journal of Psychiatry. 2003;160(3):430-7. https://doi.org/10.1176/appi.ajp.160.3.430.

54. Maire J, Galéra C, Meyer E, Salla J, Michel G. Is emotional lability a marker for attention deficit hyperactivity disorder, anxiety and aggression symptoms in preschoolers? Child Adolesc Mental Health. 2017;22(2):77-83. https://doi.org/10.1111/camh.12168.

55. Masi G, Pisano S, Milone A, Muratori P Child behavior checklist dysregulation profile in children with disruptive behavior disorders: A longitudinal study. Journal of Affective Disorder. 2015; 186: 249253. https://doi.org/10.1016/j.jad.2015.05.069.

56. Massé L, Verret C, Boudreault F. Mieux gérer sa colère et sa frustration. Montréal: Chenelière Education; 2012.

57. Massé $L$, Verreault $M$, Verret $C$. Mieux vivre avec le TDAH à la maison. Montréal: Chenelière Education; 2011.

58. McCandless S, O' Laughlin L. The Clinical Utility of the Behavior Rating Inventory of Executive Function (BRIEF) in the Diagnosis of ADHD. Journal of Attention Disorders. 2007;10(4):381-9. https://doi.org/10.1177/1087054706292115. 
59. McQuade JD, Breaux RP. Are Elevations in ADHD Symptoms Associated with Physiological Reactivity and Emotion Dysregulation in Children? J Abnorm Child Psychol. 2016. https://doi.org/10.1007/s10802-016-0227-8.

60. Melnick SM, Hinshaw SP. Emotion regulation and parenting in AD/HD and comparison boys: Linkages with social behaviors and peer preference. J Abnorm Child Psychol. 2000;28(1):73-86. https://doi.org/10.1023/a:1005174102794.

61. Mervielde I, De Fruyt F. (1999). Construction of the Hierarchical Personality Inventory for Children (HiPIC). In I. Mervielde, I. Deary, F. De Fruyt, \& F. Ostendorf, editors, Personality Psychology In Europe, Proceedings of the Eight European Conference on Personality Psychology (pp. 107-127). Tilburg, The Netherlands: Tilburg University Press.

62. Miranda A, Presentacion MJ. Efficacy of cognitive-behavioral therapy in the treatment of children with ADHD, with and without agressiveness. Psychology in the Schools. 2000;37:169-82.

63. Murray AL, Obsuth I, Zirk-Sadowski J, Ribeaud D, Eisner M. Developmental Relations Between ADHD Symptoms and Reactive Versus Proactive Aggression Across Childhood and Adolescence. Journal of Attention Disorders 2016: 1-10. https://doi.org/10.1177/1087054716666323.

64. Penton-Voak IS, Thomas J, Gage SH, McMurran M, McDonald S, Munafò MR. Increasing Recognition of Happiness in Ambiguous Facial Expressions Reduces Anger and Aggressive Behavior. Psychol Sci. 2013;24(5):688-97. https://doi.org/10.1177/0956797612459657.

65. Peyre H, Speranza M, Cortese S, Wohl M, Purper-Ouakil D, Do ADHD Children With and Without Child Behavior Checklist-Dysregulation Profile Have Different Clinical Characteristics, Cognitive Features, and Treatment Outcomes? Journal Of Attention Disorders. 2015 19(1): 63-71. https://doi.org/10.1177/1087054712452135.

66. Polanczyk G, Silva de Lima M, Lessa Horta B, Biederman J, Rohde LA. The Worldwide Prevalence of ADHD: A Systematic Review and Metaregression Analysis. American Journal of Psychiatry. 2007;164:942948. https://doi.org/10.1176/ajp.2007.164.6.942.

67. Qian Y, Chang W, He X, Yang L, Liu L, Ma Q, Li Y, Sun L, Qian Q, Wang Y. Emotional Dysregulation of ADHD in Childhood Predicts Poor Early-Adulthood Outcomes: A Prospective Follow up Study. Research in Developmental Disabilities. 2016;59:428436. https://doi.org/10.1016/j.ridd.2016.09.022.

68. Ravens-Sieberer U, Auquier P, Erhart M, Gosh A, Rajmil L, Bruil J. \& al., The Kidscreen-27 quality of life measure for children and adolescents: psychometric results from a cross-cultural survey in 13 European countries. Qual Life Res. 2007;16(8):1347-56.

69. Raysse P. (2011). Troubles du développement de l'enfant et qualité de vie familiale. Thèse de doctorat, Psychologie du développement, Université Montpellier 3, Montpellier (France).

70. Rosen PJ, Factor PI. Emotional Impulsivity and Emotional and Behavioral Difficulties Among Children With ADHD: An Ecological Momentary Assessment Study. Journal of Attention Disorders. 2015;19(9):779-93. https://doi.org/10.1177/1087054712463064.

71. Rosen PJ, Leaberry KD, Slaughter K, Fogleman ND, Walerius DM, Loren REA, Epstein JN. Managing Frustration for Children (MFC) Group Intervention for ADHD: An Open Trial of a Novel Group 
Intervention for Deficient Emotion Regulation. Cognitive Behavioral Practice. 2018. https://doi.org/10.1016/j.cbpra.2018.04.002.

72. Saini M. A meta-analysis of the psychological treatment of anger: Developing guidelines for evidence-based practice. The Journal of the American Academy of Psychiatry the Law. 2009;37(4):473-88.

73. Sánchez M, Lavigne R, Romero JF, Elósegui E. Emotion Regulation in Participants Diagnosed With Attention Deficit Hyperactivity Disorder, Before and After an Emotion Regulation Intervention. Front Psychol. 2019, 10. https://doi.org/10.3389/fpsyg.2019.01092.

74. Shaffer D, Gould MS, Brasic J, Ambrosini P, Fisher P, Bird H, Aluwhlia SA. Children's Global Assessment Scale (CGAS). Arch Gen Psychiatry. 1983;40:1228-31.

75. Shaw P, Stringaris A, Nigg J, Leibenluft E. Emotion Dysregulation in Attention Deficit Hyperactivity Disorder. American Journal of Psychiatry. 2014;171:276293. https://doi.org/10.1176/appi.ajp.2013.13070966.

76. Sjöwall D, Roth L, Lindqvist S, Thorell LB. Multiple Deficits in ADHD: Executive Dysfunction, Delay Aversion, Reaction Time Variability, and Emotional Deficits: Neuropsychological and Emotional Deficits in ADHD. Journal of Child Psychology Psychiatry. 2013;54:619627. https://doi.org/10.1111/jcpp.12006.

77. Sobanski E, Banaschewski T, Asherson P, Buitelaar J, Chen W, Franke B, Holtmann M, Krumm B, Sergeant J, Sonuga-Barke EJS, Stringaris A, Taylor E, Anney R, Ebstein RP, Gill M, Miranda A, Mulas F, Oades RD, Roeyers H, Rothenberger A, Steinhausent H-C, Faraone SV. Emotional Lability in Children and Adolescents with Attention Deficit/Hyperactivity Disorder (ADHD): Clinical Correlates and Familial Prevalence: Emotional Lability in ADHD. Journal of Child Psychology Psychiatry. 2010;51:915923. https://doi.org/10.1111/j.1469-7610.2010.02217.x.

78. Spencer TJ, Faraone SV, Surman CBH, Petty C, Clarke A, Batchelder H, Wozniak J, Biederman J. Toward Defining Deficient Emotional Self-Regulation in Children with Attention-Deficit/Hyperactivity Disorder Using the Child Behavior Checklist: A Controlled Study. Postgraduate Medicine. 2011;123(5):50-9. https://doi.org/10.3810/pgm.2011.09.2459.

79. Stoddard J, Sharif-Askary B, Harkins EA, Frank HR, Brotman MA, Penton-Voak IS, Maoz K, Bar-Haim Y, Munafo M, Pine DS, Leibenluft E. An Open Pilot Study of Training Hostile Interpretation Bias to Threat Disruptive Mood Dysregulation Disorder. J Child Adolesc Psychopharmacol. 2016;26(1):4957. https://doi.org/10.1089/cap.2015.0100.

80. Stringaris A, Vidal-Ribas P, Brotman MA, Leibenluft E. Practitioner Review: Definition, recognition, and treatment challenges of irritability in young people. J Child Psychol Psychiatry. 2018;59(7):721-39. https://doi.org/10.1111/jcpp.12823.

81. Sukhodolsky DG, Kassinove H, Gorman BS. Cognitive-behavioral therapy for anger in children and adolescents: A meta-analysis. Aggress Violent Beh. 2004;9(3):247-69. https://doi.org/10.1016/j.avb.2003.08.005. 
82. Surman CBH, Biederman J, Spencer T, Yorks D, Miller CA, Petty CR, Faraone SV. Deficient Emotional Self-Regulation and Adult Attention Deficit Hyperactivity Disorder: A Family Risk Analysis. Am J Psychiatry. 2011;168:617-23. https://doi.org/10.1176/appi.ajp.2010.10081172.

83. The KIDSCREEN Group Europe. The KIDSCREEN questionnaires Handbook. Lengerich: Pabst Science Publishers; 2006.

84. Thi Bui H, Mackie L, Hoang A, P., \& Thi Tran T. Exploring the effectiveness of cognitive behavioral therapy for Vietnamese adolescents with anger problems. Kasetsart Journal of Social Sciences. 2018: 1-5. https://doi.org/10.1016/j.kjss.2018.05.013.

85. Thompson RA, Emotion Regulation. A theme in Search of Definition. Monogr Soc Res Child Dev. $1994 ; 59(2 / 3): 25-52$.

86. Tourian L, LeBoeuf A, Breton J-J, Cohen D, Gignac M, Labelle R, Guile J-M, Renaud J. Treatment Options for the Cardinal Symptoms of Disruptive Mood Dysregulation Disorder. Journal Of The Canadian Academy Of Child Adolescent Psychiatry. 2015;24(1):41-54.

87. Vacher C, Goujon A, Romo L, Purper-Ouakil D. Efficacy of psychosocial interventions for children with ADHD and emotion dysregulation: A systematic review. Psychiatry Res. 2020; 291. https://doi.org/10.1016/j.psychres.2020.113151.

88. Van Stralen J. Emotional dysregulation in children with attention-deficit/hyperactivity disorder. ADHD Attention Deficit Hyperactivity Disorders. 2016;8(4):175-87. https://doi.org/10.1007/s12402-0160199-0.

89. Vidal-Ribas P, Brotman MA, Valdivieso I, Leibenluft E, Stringaris A. The Status of Irritability in Psychiatry: A Conceptual and Quantitative Review. Journal of the American Academy of Child Adolescent Psychiatry. 2016;55(7):556-70. https://doi.org/10.1016/j.jaac.2016.04.014.

90. Walerius DM, Reyes RA, Rosen PJ, Factor PI. Functional Impairment Variability in Children With ADHD Due to Emotional Impulsivity. Journal of Attention Disorders. 2018;22(8):724-37. https://doi.org/10.1177/1087054714561859.

91. Wang YP, Gorenstein C. Psychometric properties of the Beck Depression Inventory-II: a comprehensive review. Rev Bras Psiquiatr. 2013;35(4):416-31.

92. Wang B, Brueni LG, Isensee C, Meyer T, Bock N, Ravens-Sieberer U, Klasen F, Schlack R, Becker A, Rothenberger $A$. The Bella study group. Predictive value of dysregulation profile trajectories in childhood for symptoms of ADHD, anxiety and depression in late adolescence. European Child Adolescent Psychiatry. 2017: 1-8. https://doi.org/10.1007/s00787-017-1059-y.

93. Waxmonsky JG, Waschbusch DA, Belin P, Li T, Babocsai L, Humphery H, Pariseau ME, Babinski D, Hoffman MT, Haak JL, Mazzant JA, Fabiano GA, Pettit JW, Fallahazad N, Pelham WE. A Randomized Clinical Trial of an Integrative Group Therapy for Children With Severe Mood Dysregulation. Journal of the American Academy of Child Adolescent Psychiatry. 2016;55:196207. https://doi.org/10.1016/j.jaac.2015.12.011.

94. Webster-Stratton C. The Incredible Years: A trouble-shooting guide for parents of children ages 3-8 years. Seattle: Incredible Years Press; 2006. 
95. Wehmeier PM, Schacht A, Barkley RA. Social and Emotional Impairment in Children and Adolescents with ADHD and the Impact on Quality of Life. Journal of Adolescent Health. 2010;46:209217. https://doi.org/10.1016/j.jadohealth.2009.09.009.

96. White BA, Jarrett MA, Ollendick TH. Self-Regulation Deficits Explain the Link between Reactive Aggression and Internalizing and Externalizing Behavior Problems in Children. Journal of Psychopathology Behavioral Assessment. 2013;35(1):1-9. https://doi.org/10.1007/s10862-0129310-9.

97. Winters DE, Fukui S, Leibenluft E, Hulvershorn LA. Improvements in Irritability with Open-Label Methylphenidate Treatment in Youth with Comorbid Attention Deficit/Hyperactivity Disorder and Disruptive Mood Dysregulation Disorder. Journal Of Child Adolescent Psychopharmacology. 2018;28(5):298305. https://doi.org/10.1089/cap.2017.0124.

98. Zwi M, Jones H, Thorgaard C, York A, Dennis JA. Parent training interventions for Attention Deficit Hyperactivity Disorder (ADHD) in children aged 5 to 18 years. Cochrane Database of Systematic Reviews. 2011. https://doi.org/10.1002/14651858.CD003018.pub3. 